\title{
Azelainsäure, ein weiterer Bestandteil des menschlichen Harns
}

\author{
Von H. R. Greub und K. Bernhard \\ Aus dem Physiologisch-chemischen Institut der Universität Basel (Vorsteber: Prof. Dr. K. Bernbard)
}

(Eingegangen am 13. März 1967)

Aus normalem Menschenharn und aus Hundeharn wurde Azelainsäure isoliert. Gesunde Versuchspersonen scheiden etwa $18-28 \mathrm{mg}$ Azelainsäure pro Tag aus, Hunde von $25 \mathrm{~kg}$ Körpergewicht bei normalem Futter 10-15 mg. Rattenharn enthält keine Azelainsäure.

Azelcic acid was isolated from normal human urine and dog urine. Healthy experimental persons excrete about $18-28 \mathrm{mg}$. of azeleic acid per day. Dogs weighing $25 \mathrm{~kg}$., on a normal diet, excrete $10-15 \mathrm{mg}$. per day. Rat's urine contains no azeleic acid.

Außer der schon vor langem nachgewiesenen Oxalsäure wurden weitere Dicarbonsäurë̈ als Bestandteile von normalem Menschenharn oder von solchem verschiedener Karnivoren und Herbivoren festgestellt. FLASCHENTRÄGER und BERNHARD (1) fanden im Menschenharn pro Tag 3-5 mg Furan-dicarbonsäure, nach WeITZEL (2) kommt auch Bernsteinsäure darin regelmäßig vor. H. Müller (3) gewann Azelain- und Pimelinsäure aus Kuhharn. LEDERER und Mitarbeiter (4) erhielten bei der Gewinnung steroider Verbindungen aus dem Harn schwangerer Stuten pro Liter $13 \mathrm{mg}$ Azelainsäure. Verabreichung von Triundecylin führte bei Versuchspersonen zur Ausscheidung kleiner Mengen von Nonandicarbonsäure, Azelainsäure und Pimelinsäure (5). Mensch und Hund scheiden auch kleine Mengen aufgenommener Azelainsäure im Harn größtenteils wieder aus (6). In früheren Versuchen (7) stellten wir nach Gaben D-signierter Azelainsäure an gesunde Versuchspersonen eine Verdünnung des Deuterium-Gehaltes bei der aus dem Harn zurückgewonnenen Säure fest von z. B. 7,85 auf 7,70 bzw. 5,31 auf 5,04, was darauf hindeutet, daß Azelainsäure im Stoffwechselgeschehen offensichtlich in kleinen Mengen gebildet wird.

Wir konnten nun mit chromatographischen Methoden aus normalem Menschenharn und aus Hundeharn Azelainsäure isolieren. Durch Gaschromatographie der veresterbaren und über einer Silicagel-Kolonne gereinigten Anteile der ätherischen Harnextrakte lassen sich, wie aus den Tabellen 1 und 2 hervorgeht, quantitative Angaben über den Gehalt an Azelainsäure machen. Über ihre Herkunft sind nur Vermutungen möglich. Im Hundefutter ist sie nicht vorhanden und wohl auch nicht in der menschlichen Nahrung, Die Annahme, Ölsäure könnte durch Aufspaltung der Doppelbindung Azelainsäure geben, ist naheliegend, aber nicht bewiesen.

Aus Rattenharn konnten wir diese Dicarbonsạure nicht erhalten; die Gaschromatogramme verschiedener Harnproben ließen keine Azelainsäure erkennẹn. Wurḍe letztere an Ratten im Ausmaße von $1 \mathrm{~g} / \mathrm{kg} / \mathrm{Tag}$ verfüttert, ließen sich aus dem Harne rund $40 \%$ wiedergewinnen.

\section{Methodik}

Die nach Ansäuern des Harnes mit Äther erhaltenen Extrakte wurden mit 12-proz. methanol. $\mathrm{BF}_{3}$-Lösung verestert, indem $z$. B. $500 \mathrm{mg}$ Harnextrakt in wenig Methanol gelöst mit $10 \mathrm{~m} /$ methanol. Bortrifluoridlösung erwärmt wurden. Das Estergemisch, von dem anzunehmen ist, es enthalte alle im Harn vorhandenen Dicarbon-
Tab. 1

Azelainsäure im Menschen-Harn

\begin{tabular}{|c|c|c|c|c|c|c|}
\hline \multicolumn{3}{|c|}{ Versuchspersonen } & \multirow[b]{2}{*}{$\mathrm{ml}$} & \multicolumn{3}{|c|}{ Harn von 24 Stunden } \\
\hline & Alter & Geschlecht & & $\begin{array}{l}\text { g Äther- } \\
\text { extrakt }\end{array}$ & $\begin{array}{c}\% \text { bez. auf } \\
\text { Ätherextrakt }\end{array}$ & $\begin{array}{l}\text { total } \\
\mathrm{mg}\end{array}$ \\
\hline$M$ & 25 & m & 1500 & 2,76 & 0,70 & 19,4 \\
\hline E & 27 & $\mathrm{~m}$ & 880 & 2,62 & 0,93 & 24,3 \\
\hline $\begin{array}{l}\mathbf{G} \\
\mathbf{G}\end{array}$ & $\begin{array}{l}28 \\
28\end{array}$ & $\underset{\mathrm{m}}{\mathrm{m}}$ & $\begin{array}{l}1500 \\
1550\end{array}$ & $\begin{array}{l}1,80 \\
1,13\end{array}$ & $\begin{array}{l}1,37 \\
2,24\end{array}$ & $\begin{array}{l}24,8 \\
25,3\end{array}$ \\
\hline $\mathbf{G}$ & 28 & m & 1510 & 1,19 & 1,56 & 18,7 \\
\hline $\mathrm{K}$ & 31 & $\mathrm{~m}$ & 1200 & 2,66 & 0,74 & 19,2 \\
\hline B & 63 & $\mathrm{~m}$ & 1330 & 2,58 & 1,07 & 27,5 \\
\hline $\mathbf{s}$ & 26 & $w$ & 1180 & 3,26 & 0,57 & 18,4 \\
\hline $\mathrm{J}$ & 30 & $w$ & 1180 & 1,77 & 1,20 & 21,4 \\
\hline $\mathrm{F}$ & 31 & w & 1150 & 2,48 & 1,03 & 25,8 \\
\hline$\overline{\mathbf{x}}$ & & & 1310 & 2,22 & 1,14 & 22,5 \\
\hline
\end{tabular}

Tab. 2

Azelainsäure im Hunde-Harn

\begin{tabular}{|c|c|c|c|c|c|}
\hline \multicolumn{2}{|c|}{ Tier } & \multicolumn{4}{|c|}{ Harn von 24 Stunden } \\
\hline Nr. & $\underset{\mathbf{k g}}{\underset{\text { Gewicht }}{ }}$ & $\mathrm{ml}$ & $\begin{array}{l}\text { g Äther- } \\
\text { extrakt }\end{array}$ & $\begin{array}{l}\% \text { bez. auf } \\
\text { Âtherextrakt }\end{array}$ & $\begin{array}{l}\text { total } \\
\text { mg }\end{array}$ \\
\hline 1 & 25 & 910 & 0,40 & 2,9 & 11,8 \\
\hline 1 & & 500 & 0,43 & 2,7 & 11,5 \\
\hline 1 & & 550 & 0,58 & 1,7 & 10,0 \\
\hline 2 & 25 & 480 & 0,61 & 2,0 & 12,4 \\
\hline 2 & & 450 & 0,47 & 3,0 & 14,1 \\
\hline 2 & & 590 & 0,56 & 2,8 & 15,4 \\
\hline
\end{tabular}

Tier $1: \bar{x}=11,1 \mathrm{mg} ;$ Tier $2: \bar{x}=14,0 \mathrm{mg}$.

säuren, wies aufgrund der chromatographischen Analyse z. B. $20 \%$ Azelainsäure-Dimethylester auf. Wir brachten $500 \mathrm{mg}$ davon zur Abtrennung von Begleitstoffen mit wenig Äther auf eine Säule ( $2 \mathrm{~cm}$ Durchmesser, $10 \mathrm{~cm}$ Höhe), enthaltend $15 \mathrm{~g}$ Kieselgel Merck $(0,05-0,2 \mathrm{~mm})$ und eluierten anschließend mit $150 \mathrm{ml}$ Äther: Petroläther (2:3). Eine zweite Reinigung erfolgte wieder mit einer Kieselgelkolonne $(35 \mathrm{~g}$, Durchmesser $3 \mathrm{~cm}$, Höhe $38 \mathrm{~cm}$ ), welche mit $200 \mathrm{mg}$ Estergemisch gelöst in wenig Äther beschickt wurde. Zur Eluierung diente ein Lösungsmittelgemisch von 9 Teilen Benzol, 4 Teilen Petroläther und je 1 Teil Äther und Aceton. Das abfließende Eluat wurde im Fraktionensammler aufgefangen und durch Dünnschicht-Chromatographie geprüft (Schicht Kieselgel G neutral, Fließmittel wie oben, Entwicklung mit $\mathrm{J}_{2}$-Dämpfen, braune Flecken auf hellgelbem Grund $\left(R_{\mathrm{F}}\right.$-Wert für Azelainsäure-Methylester 0,63). Die maximale Anreicherung an Azelainsäure-Methylester betrug etwa 40\%. Wir haben solche Anteile vereinigt und ciner Molekular-Destillation unterworfen. Aus der Hauptfraktion gewannen wir mittels eines Gaschromatographen für präparative Zwecke unter Verwendung einer Kolonne aus Hyflo Supercell und Bernsteinsäure-Polyäthylester, Fraktionen von weitgehend reinem Azelainsäure-Methylester. Sie wurden verseift und nach Ansäuern die freie Säure mit Äther-Heptan extrahiert. Nach Abdampfen des Lösungsmittels rèsultierte aus ÄtherHeptan umkristallisiert reine Azelainsäure, Fp. 106-107 ${ }^{\circ}$, MischFp. $106-107^{\circ}$. 
Alialyse:

$\mathrm{C}_{9} \mathrm{H}_{16} \mathrm{O}_{4}$ (Mol.-Gew. 188,22) ber.: C 57,40 H 8,57

gef: $57,34 \quad 8,51$ (aus Mènschenharn)

57,67 8,57 (aus Hundeharn)

Die Mikrotitration ergab ein Äquivalentgewicht von 93,9 und 93,8 (ber.: 94,11).

Die Ausbeuten an bei dieser Aufarbeitung erhaltenen Fraktionen sind aus der Tabelle 3 ersichtlich.

\section{Fïtterung won Azelainsäure an Ratten}

Fünf männliche Ratten (Körpergewicht im Mittel $430 \mathrm{~g}$ ) erhielten während 12 Tagen einem normalen Futter 25,6 g Azelainsäure als Na-Salz beigemischt. Die Harnmenge von $1700 \mathrm{ml}$, bei saurer Reaktion mit Äther er-
Tab. 3

Isolierung von Azelainsäure aus Menschen- und Hundeharn

\begin{tabular}{lcc}
\hline & Mensch & Hund \\
\hline Harnmenge in l & 12,0 & 6,4 \\
Áther-Extrakt in g & 9,5 & 5,6 \\
Methylester & 1,4 & 0,85 \\
nach 1. Reinigung, g & 0,29 & 0,17 \\
nach 2. Reinigung, g & 170 & 110 \\
nach Molekular-Destillation mg & 80 & 50 \\
nach präp. Gaschromatographie & 25 & 15 \\
Azelainsäure, rein mg & & \\
\hline
\end{tabular}

schöpfend extrahiert, lieferte 13,27 g:Rohextrakt. Daraus konnten 7,20 g rohe Azelainsäure, nach Umkristallisierung $5,87 \mathrm{~g}$ reine Azelainsäure, Fp. $107-108^{\circ}$ erhalten werden. Der restliche Rohextrakt und die Mutterlauge enthielten laut chromatographischer Bestimmung weitere 4,22 g Azelainsäure.

\section{Literatur}

1. Flaschenträger, B. und K. Bernhard, Z. physiol. Chem. 246, 124 (1937). - 2. Wertzel, G., Z. physiol. Chem. 282, 174 (1947). - 3. MülLER, J. H., J. biol. Chem. 119, 121 (1937). 4. Lederer, E. und J. Polonsky, Biochem. Biophys. Acta 2, 431 (1948). - 5. Verkade, P. E., M. Elzas, J. VAN DER LeE,
H. H. De Wolff, A. Verkade-Sandiergen und D. van der SANDE, Z. physiol. Chem. 215, 225 (1933). - 6. Bernhard, K., Z. physiol. Chem. 246, 133 (1937). - 7. Bernhard, K. und U. Gloor, Helv. Chim. Acta 35, 608 (1952).

Prof. Dr. phil. K. Bernhard CH 4051 Basel, Vesalgasse 1

\section{KURZMITTEILUNGEN}

\section{Serumzinkbestimmung durch Atomabsorptionsspektrometrie}

Von Th. HaAs, G. Lehnert und K. H. SchalleR

Aus dem Institut fiir Arbeits- und Sozial-Medizin der Universität Erlangen-Niirnberg (Direktor: Prof. Dr. med. H. Valentin)

(Eingegangen am 20. April 1967)

Die medizinische Forschung der letzten Jahre läßt deutlich erkennen, daß zukünftig den Arzt Krankheitserscheinungen unter dem Bild von Hypermetallosen, Hypometallosen oder Dysmetallosen intensiver als bisher beschäftigen werden. Eine Vervollkommnung, Verfeinerung und Vereinfachung der Spurenelementanalyse im biologischen Material für die Belange der Praxis ist daher unumgänglich. Hierzu bietet die Atomabsorpticnsspektrometrie („AAS“) alle Voraussetzungen. - Nachfolgend möchten wir über Erfahrungen berichten, die wir bei der Erarbeitung einer atomabsorptionsspektometrischen Bestimmungsmethode für $Z$ ink im Serum machen konnten.

Serumzinkanalysen mit Hilfe der AAS wurden in der Literatur wiederholt beschrieben. Nach HoNEGGER löst man das eiweißgebundene Zink durch Zusatz von $6 \mathrm{~N}-$ Salzsäure heraus, enteiweißt, zentrifugiert und mißt das Zentrifugat gegen ein Modellserum mit variiertem Zinkgehalt. - Eine Methode, bei der das Plasma nach vorangehender Gefriertrocknung ebenfalls enteiweißt wird, wurde von Prasad und Mitarbeitern angegeben.

Die direkte atomabsorptionsspektrometrische Messung von nicht enteiweißten Seren ist nur in verdünntem Zustand möglich. Hierzu werden Geräte hoher Empfindlichkeit benötigt. Fuwa und Mitarbeiter erreichen dies durch Einsatz von Absorptionsröhren. Damit konnten 1:10 verdünnte Seren ausreichend genau gegen wäßrige Zinkstandardlösungen gemessen werden. Derartige Geräte befinden sich jedoch zur Zeit nicht im Handel. Nach SPRAGUE und Slavin ist auch am Perkin-Elmer-Gerät, Modell 303, die direkte Messung von 5:10 verdünnten Seren gegen wäßrige Standardlösungen möglich.

\section{Methodik}

Als Atomabsorptionsspektrometer wurde von uns das Gerät der Fa. Beckman, ausgerüstet mit einem Turbulenzbrenner, in Kombination mit dem Spektralphotometer DU verwendet.

\section{Apparative Arbeitsbedingungen}

Zn-Hohlkathodenlampe (Quarzlampengesellschaft Hanau); Stromstärke: $5 \mathrm{~mA}, 3$ Brenndüsen, Flamme dreifach durchstrahlt; Luftdruck: $11 \mathrm{psi}$, Wasserstoffdruck: $4 \mathrm{psi}$, Spaltbreite: $0,05 \mathrm{~mm}$, Wellenlänge: 2138,6 ; Schreiber: 10"-Recorder, Fa. Beckman. Damit konnte eine Nachweisgrenze von $0,47 \mu \mathrm{g} \% \mathrm{Zn}$, gemessen bei $1 \%$ Absorption, erreicht werden. Bezüglich näherer Angaben zur Zinkbestimmung mit dem verwendeten Gerät verweisen wir auf unsere Darstellung im Beckman-Report ${ }^{1}$ ).

Unsere direkten Messungen eines Serums gegen wäßrige Eichlösungen erwiesen sich nicht frei von Interferenzen. $\mathrm{Da}$ der hohe Salz- und Eiweißgehalt des Serums die Sprühgeschwindigkeit des Brenners verringert, wird die Absorption um etwa 30\% erniedrigt. Verdünnt man das Serum in einen Bereich von $1: 10$ bis $5: 10$, so findet keine Absorptionserniedrigung statt, da dann die Sprühgeschwindigkeit den Eichlösungen angeglichen ist. - An weiteren Interferenzen zeigte sich eine unspezifische heterochromatische Absorption durch Serumelektrolyte. Sie entsteht durch Streuung des Lichts der Hohlkathodenlampe, ausgehend von soliden Salzpartikeln innerhalb der Flamme. So verursachte ein zinkfreies Modellserum (Na-, K-, Ca-Gehalt entsprechend dem Serumspiegel) eine durchschnittliche Absorption von 0,035. Für diese Fremdabsorption sind in erster Linie Kochsalzpartikel verantwortlich. Die von Fuwa und Mitarbeitern beschriebene Absorptionserniedrigung wäßriger Zinklösungen durch Natriumphosphat konnte von uns ebenfalls beobachtet werden. Sie trat bereits bei Konzentrationen von weniger als $0,001 \mathrm{M}$ Natriumphosphat ein. Die Absorption von Zinklösungen innerhalb eines Modellserums

1) HaAs, Th., G. Lehnert und K. H. Schaller, Atomabsorptionsspektrometrische Zinkbestimmung im Serum. Beckman Report, im Druck. 